\title{
Using a Robust Design Approach to Optimize Chair Set-up in Wheelchair Sport ${ }^{+}$
}

\author{
David S. Haydon ${ }^{1, *}$, Ross A. Pinder ${ }^{2}$, Paul N. Grimshaw ${ }^{1}$ and William S. P. Robertson ${ }^{1}$ \\ 1 Mechanical Engineering, University of Adelaide, Adelaide 5005, Australia; \\ paul.grimshaw@adelaide.edu.au (P.N.G.); will.robertson@adelaide.edu.au (W.S.P.R.) \\ 2 Australian Paralympic Committee, Sydney 2127, Australia; ross.pinder@paralympic.org.au \\ * Correspondence: David.haydon@adelaide.edu.au; Tel.: +61-8-8313-5460 \\ + Presented at the 12th Conference of the International Sports Engineering Association, Brisbane, Queensland, \\ Australia, 26-29 March 2018.
}

Published: 11 February 2018

\begin{abstract}
Optimisation of wheelchairs for court sports is currently a difficult and time-consuming process due to the broad range of impairments across athletes, difficulties in monitoring on-court performance, and the trade-off set-up that parameters have on key performance variables. A robust design approach to this problem can potentially reduce the amount of testing required, and therefore allow for individual on-court assessments. This study used orthogonal design with four set-up factors (seat height, depth, and angle, as well as tyre pressure) at three levels (current, decreased, and increased) for three elite wheelchair rugby players. Each player performed two maximal effort sprints from a stationary position in nine different set-ups, with this allowing for detailed analysis of each factor and level. Whilst statistical significance is difficult to obtain due to the small sample size, meaningful difference results aligning with previous research findings were identified and provide support for the use of this approach.
\end{abstract}

Keywords: wheelchair rugby; wheelchair design; propulsion; kinematics; on-court testing; maximal acceleration

\section{Introduction}

The optimisation of wheelchair sport performance has three levels at which it occurs: (i) the athlete, where the focus is on physiological performance and skill execution; (ii) the wheelchair itself, where minimising detrimental factors such as rolling resistance provides performance benefits; and (iii), the athlete-user interface, which focuses on the interaction between the athlete and wheelchair [1]. Wheelchair design has been repeatedly shown to influence propulsion in both daily living and sport [2,3], with these design parameters including seat height, depth, and angle [2,4], as well as camber [5] and wheel diameter [6]. However, the selection of optimal settings for these parameters is difficult due to the trade-off between performance factors, and the effects of these parameters being highly individualised due to differences in physical activity limitations of athletes in the sport. Players are eligible to participate in wheelchair rugby (WCR) if they have impairments including impaired muscle power or limb deficiencies affecting multiple limbs [7]. The level of physical activity limitation is then accounted for using a classification system, where points are allocated from 0.5-3.5 points based on the strength, range of motion, and co-ordination (referred to as 'function') of the trunk, arm, and hand [8]. Players with different impairments, and varying capabilities, can therefore be assigned similar classification scores. This requires individual assessments of wheelchair set-up, as set-up parameters have varying effects on players despite similar classification scores. Seat height, for example, can be lowered to provide greater access to the pushrim and wheel, increasing time for force application [9]. However, lowering the seat height 
potentially limits ball handling capabilities which is clearly undesirable in court sports. Depending on capabilities of the player, including amount of trunk function, varying levels of pushrim access are possible with the same seat height. Similar trade-off effects exist for most parameters; e.g., seat depth - a deeper seat position can improve stability but limit agility [10]; seat angle - an increase provides greater stability, but limits trunk mobility and therefore acceleration from standstill [4]. Other factors, such as tyre pressure, have also been suggested to influence performance due to the effects on tyre grip and wheel spin, although this has yet to be investigated.

To address the need for improved individualised wheelchair prescription, the use of a robust design approach to assess the effects of specific configuration parameters has previously been investigated [11]. This focused on the effects of wheel diameter, camber angle, seat height, and seat depth and the ability to maximise push phase velocity and the mean acceleration of the push as well as minimising the recovery time. While these measures can potentially provide a benefit to performance, testing was performed on an ergometer, with this approach repeatedly shown to alter propulsion kinematics compared with overground propulsion [12]. Recent advancements in technology have improved the capabilities of monitoring on-court activity profiles through the use of tracking systems or inertial measurement units (IMUs) [13]. In addition, previous approaches [5,6,11] have not monitored kinematics throughout testing and no assessment of the final recommended setting to confirm the validity of the approach performed. It therefore remains unclear whether a robust design approach can provide an improved approach to individualised wheelchair design. This study used orthogonal design to investigate a range of wheelchair design parameters and their effects on sprint time and propulsion kinematics during an on-court linear sprint from standstill.

\section{Materials and Methods}

Three elite WCR players (age: $27.7 \pm 4$ years; international experience: $5.7 \pm 4$ years, classifications of 1.0- (Player 1), 2.0- (Player 2), and 3.5-points (Player 3)) provided written, informed consent before completing testing. An adjustable rugby wheelchair was used throughout on-court testing, with seat height (SH), seat depth (SDep), seat angle (SA), and tyre pressure (TP) the set-up factors varied (Figure 1). All other configuration parameters were kept constant throughout testing. An L9 orthogonal array was implemented, allowing the four factors to be investigated at three levels; the player's current set-up (i), and both an increase (ii) and decrease (ii) to the parameter. For $\mathrm{SH}$ and SDep, these were by increments of $\pm 15 \mathrm{~mm}, \mathrm{SA}$ by $\pm 5^{\circ}$, and TP by \pm 15 psi. The order in which the nine set-ups were tested was randomised, and a setting similar to their current wheelchair set-up (in terms of SH, SDep and SA) incorporated without notifying the player of the set-up during testing.

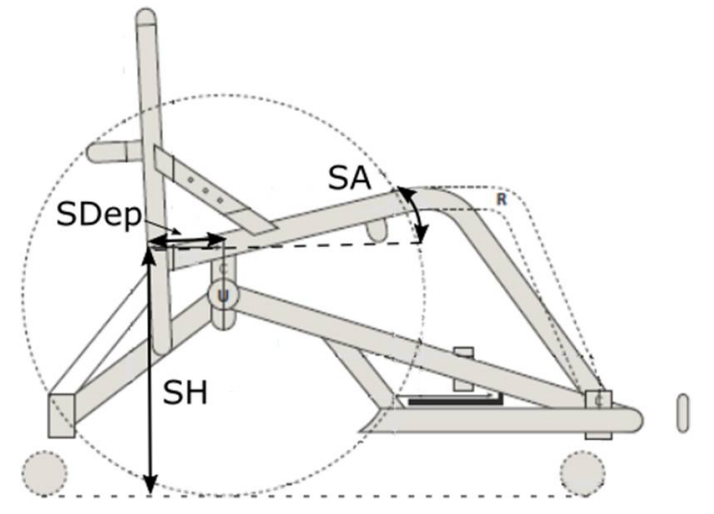

Figure 1. During testing, SH, SDep, and SA were all adjusted as shown, as well as TP (not shown). Figure adapted from [14].

For each configuration, players were instructed to familiarise themselves with the set-up by pushing around the court in a manner similar to their usual warm-up procedure. Once the player was comfortable in the set-up, they then completed two $5 \mathrm{~m}$ sprints from a stationary position initiated in their own time. This test focused on the ability to accelerate from standstill, previously 
identified as a crucial factor for performance $[4,10]$, and the resultant kinematic changes. Time to complete each test was monitored using laser timing gates (Kinematic Measurement System, Fitness Technology, Australia), with players given sufficient break between tests to minimise any effects of fatigue. Players provided feedback for each set-up detailing their perception of key performance factors, without knowledge of performance times.

Throughout testing, IMUs (500 Hz, IMeasureU, Auckland, New Zealand) were attached to the centre-front of the frame, and each wheel near the axle to avoid interference during the stroke phase. The IMU on the frame allowed monitoring of the overall accelerations of the chair, while the wheel located IMUs allowed for detection of individual left and right contacts. The frame IMU data was low-pass filtered at $20 \mathrm{~Hz}$ (Butterworth filter, order 5, bidirectional, $-6 \mathrm{~dB}$ cutoff frequency), similar to previous work in high-intensity wheelchair testing [13], before being used for analysis of intra-stroke acceleration profiles. For the $5 \mathrm{~m}$ sprints, video $(120 \mathrm{~Hz}$, GoPro Hero3+, GoPro, San Mateo, CA, USA) was recorded from rear and side views, and synchronised with IMU data. This synchronisation was performed using a sharp strike to the frame of the wheelchair, which caused a large peak in the acceleration data from the IMU. This strike was also evident in each video, with the corresponding frame selected to match the instance of the acceleration peak. Contact and release points for the first three strokes were then identified by viewing and selecting key points in the frame and wheel acceleration data using a custom MATLAB (version R2016a) code. The expected contact or release position in the acceleration traces was selected, which prompted the corresponding video frame from the side (i.e., left or right) and back view- as well as two frames before and after-to be viewed and the video frame corresponding to the event selected. If the desired frame was not visible, the user was asked to re-select a point in the acceleration trace. Contact (ContAng) and release (RelAng) angles of the hand in the respective frames were then calculated by selecting the centre of the wheel, top dead centre (TDC), and the hand location on the wheel [15]. This process allowed for the determination of stroke times (StrokeTime), recovery times (RecTime), and cycle times (CycTime) for each individual stroke.

The effect of specific configuration parameters was then assessed using the mean responses for each level of the factor (e.g., average of all sprint times that involved a decrease to the $\mathrm{SH}$ ). Greatest emphasis was given to the sprint times due to their direct relevance to on-court performance based on acceleration from standstill being a key performance factor. Due to the low sample sizes for each factor level, statistical significance is hard to achieve. Therefore, the typical error (TE) was calculated, with upper and lower limits then used to assess meaningful differences between factor levels $( \pm 1 \mathrm{TE}$ for sprint times, $\pm 1.5 \mathrm{TE}$ for propulsion kinematics). For Player 1 , with a mean ( \pm standard deviation) sprint time of $2.58 \pm 0.07 \mathrm{~s}$, this meant lower and upper limits were $2.53 \mathrm{~s}$ and $2.63 \mathrm{~s}$ for meaningful differences, respectively. Player 2 (mean $2.38 \pm 0.17 \mathrm{~s}$ ) had limits of $2.26 \mathrm{~s}$ and $2.50 \mathrm{~s}$, while Player 3 $(1.83 \pm 0.05 \mathrm{~s})$ had limits of $1.79 \mathrm{~s}$ and $1.87 \mathrm{~s}$.

\section{Results}

Mean sprint times for each player, factor, and level are presented in Table 1. Using the TE to identify meaningful differences, Player 1's performance improved with a reduced SDep, and Player 2 's performance improved with a reduced SA. Both of these examples also displayed small standard deviations, indicating that this improvement was consistent across all set-ups with these factor levels. Surprisingly, the current TP for Player 3 potentially decreased performance, with the mean sprint time matching the upper limit from TE calculations. The selection of a theoretical best set-up for a $5 \mathrm{~m}$ sprint can be identified by selecting the factor level that produced the fastest sprint times and combining these. For Player 1, this would mean a set-up with decreased SH, SDep, and SA, with TP appearing to have little influence. 
Table 1. Mean (Standard Deviation) sprint times (in seconds) for each player, factor, and level (Current-Cur; Decrease-Dec; Increase-Inc.) from testing.

\begin{tabular}{|c|c|c|c|c|c|c|c|c|c|c|c|c|}
\hline \multirow{2}{*}{ Player } & \multicolumn{3}{|c|}{ Seat Height } & \multicolumn{3}{|c|}{ Seat Depth } & \multicolumn{3}{|c|}{ Seat Angle } & \multicolumn{3}{|c|}{ Tyre Pressure } \\
\hline & Dec & Cur & Inc. & Dec & Cur & Inc. & Dec & Cur & Inc. & Dec & Cur & Inc. \\
\hline 1 & $\begin{array}{c}2.56 \\
(0.07)\end{array}$ & $\begin{array}{c}2.60 \\
(0.09)\end{array}$ & $\begin{array}{c}2.59 \\
(0.05)\end{array}$ & $\begin{array}{c}2.52 \\
(0.02)\end{array}$ & $\begin{array}{c}2.58 \\
(0.06)\end{array}$ & $\begin{array}{c}2.64 \\
(0.04)\end{array}$ & $\begin{array}{c}2.54 \\
(0.06)\end{array}$ & $\begin{array}{c}2.60 \\
(0.05)\end{array}$ & $\begin{array}{c}2.61 \\
(0.08)\end{array}$ & $\begin{array}{c}2.58 \\
(0.09)\end{array}$ & $\begin{array}{c}2.58 \\
(0.05)\end{array}$ & $\begin{array}{c}2.59 \\
(0.08)\end{array}$ \\
\hline 2 & $\begin{array}{c}2.38 \\
(0.16) \\
\end{array}$ & $\begin{array}{c}2.47 \\
(0.24) \\
\end{array}$ & $\begin{array}{c}2.29 \\
(0.06) \\
\end{array}$ & $\begin{array}{c}2.32 \\
(0.16) \\
\end{array}$ & $\begin{array}{l}20.39 \\
(0.22) \\
\end{array}$ & $\begin{array}{c}2.42 \\
(0.18) \\
\end{array}$ & $\begin{array}{c}2.21 \\
(0.02) \\
\end{array}$ & $\begin{array}{c}2.45 \\
(0.17) \\
\end{array}$ & $\begin{array}{c}2.47 \\
(0.12) \\
\end{array}$ & $\begin{array}{c}2.36 \\
(0.20) \\
\end{array}$ & $\begin{array}{c}2.32 \\
(0.13) \\
\end{array}$ & $\begin{array}{c}2.45 \\
(0.20) \\
\end{array}$ \\
\hline 3 & $\begin{array}{c}1.85 \\
(0.07)\end{array}$ & $\begin{array}{c}1.80 \\
(0.03)\end{array}$ & $\begin{array}{c}1.83 \\
(0.03)\end{array}$ & $\begin{array}{c}1.83 \\
(0.09)\end{array}$ & $\begin{array}{c}1.83 \\
(0.03)\end{array}$ & $\begin{array}{c}1.82 \\
(0.03)\end{array}$ & $\begin{array}{c}1.81 \\
(0.02)\end{array}$ & $\begin{array}{c}1.86 \\
(0.07)\end{array}$ & $\begin{array}{c}1.81 \\
(0.04)\end{array}$ & $\begin{array}{c}1.81 \\
(0.04)\end{array}$ & $\begin{array}{c}1.87 \\
(0.05)\end{array}$ & $\begin{array}{c}1.80 \\
(0.01)\end{array}$ \\
\hline
\end{tabular}

In addition to assessment of sprint time, the kinematics (ContAng, RelAng, and StrokeAng) were investigated for the first three strokes of the sprint. Exemplar data for Player 3 is presented in Figure 2 for each factor level (represented by a bar on each 'wheel'). Although not reaching the meaningful threshold, reducing SA shows a trend towards slight increases in RelAng for all strokes. A similar trend is evident for reduced SDep, while reducing SH may increase the ContAng for strokes one and two. Player 1 displayed meaningful reductions in ContAng for strokes two and three when reducing SA, as well as larger RelAngs for the same strokes. ContAngs also displayed a non-meaningful trend towards smaller ContAngs with increasing SH. Visual inspection showed that Player 2 used smaller RelAngs for all strokes when the SA was increased, despite not reaching limits for meaningful difference.

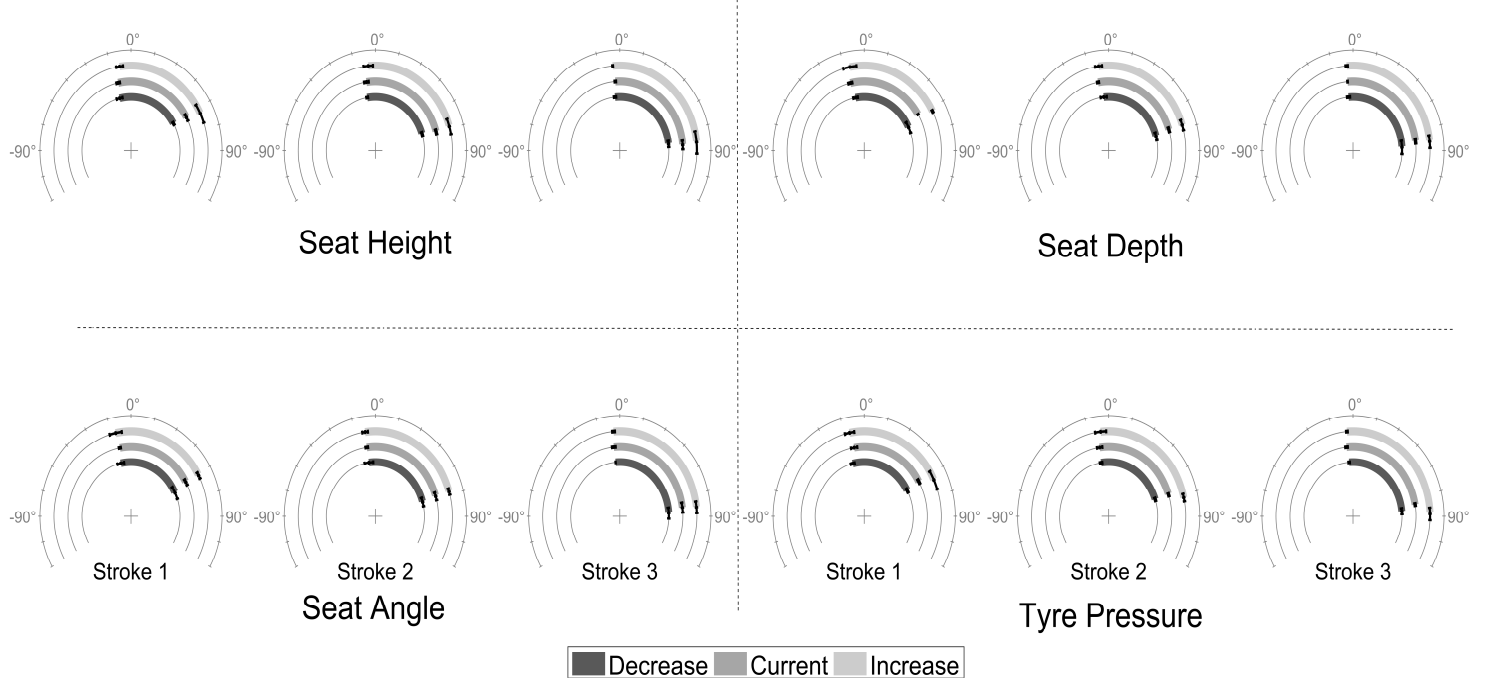

Figure 2. The ContAng, RelAng, and StrokeAng for each factor and level are presented for the first three strokes for Player 3. Each row represents a single factor, with the current, decreased, and increase to the specific factor on each 'wheel'.

StrokeTimes and CycTimes for Player 2 decreased across all strokes with an increased SH $(0.68$ $\pm 0.04 \mathrm{~s}, 0.34 \pm 0.03 \mathrm{~s}, 0.28 \pm 0.01 \mathrm{~s}$ for StrokeTimes and $0.95 \pm 0.04 \mathrm{~s}, 0.59 \pm 0.02 \mathrm{~s}, 0.51 \pm 0.02 \mathrm{~s}$ for CycTimes for increased SH compared with $0.70 \pm 0.09 \mathrm{~s}, 0.36 \pm 0.03 \mathrm{~s}, 0.29 \pm 0.02 \mathrm{~s}$ for StrokeTimes and $0.96 \pm 0.08 \mathrm{~s}, 0.61 \pm 0.04 \mathrm{~s}, 0.53 \pm 0.03 \mathrm{~s}$ for CycTimes for overall averages). Similar findings were evident for Player 3 with increased SH, although Player 1 displayed no clear trends for StrokeTime, RecTime or CycTime across any factors involved in testing.

\section{Discussion}

The assessment of rugby wheelchair set-ups during on-court testing has previously been limited, largely due to the individualised effects relating to the range of physical activity limitations in WCR, difficulty in assessing relevant on-court activity, and time and cost constraints. This approach investigated the ability of using an adjustable rugby wheelchair and an orthogonal design approach 
on the effects of an on-court $5 \mathrm{~m}$ sprint and the corresponding propulsion kinematics. Case-study approaches, such as those presented, are required as group analyses are likely to mask important individual findings, largely due to the variations in athlete impairments and activity limitation.

Although significant differences were not investigated due to the low sample sizes for each factor level and player, TE calculations provided an approach for assessing meaningful differences. Differences were evident for improved sprint time for decreased SDep for Player 1 and decreased SA for Player 2. There was also a potential detrimental effect using the current TP for Player 3, although this matched the upper limit set from TE calculations.

As previous research has described, changes to wheelchair set-up cause alterations to the propulsion approach $[9,10]$. This is supported by Figure 2, where there are visible differences in the ContAng and RelAng for Player 3 when changing important set-up parameters, including increases to RelAngs when reducing SA. This result is supported by previous literature, with reduced SAs previously been shown to and promote greater trunk range of motion, as well as altering the trunk position for the first stroke [4]. This also likely explains the ContAngs closer to TDC found for the decreased SA in Player 1, as the trunk position promotes a forward 'shift' in the stroke region around the wheel. Similar conclusions can be made for the slight increases in all RelAngs for decreased SDep in Players 1 and 3. A smaller SDep allows the player to reach further forward relative to the wheel axle, hence promoting increased RelAngs. Due to relatively small kinematic changes, the anecdotal evidence from those experienced in the sport remains important in confirming key findings [16]. Player responses following each set-up aided in the assessment of kinematic changes, as comments often reflected how the set-up was perceived to position them relative to the wheels.

Decreases in StrokeTime and CycTime for Players 2 and 3 also aligned with expectations, with increasing SH reducing the access to the pushrim/wheel for the player [9]. These results are somewhat surprising, as Player 1 was also expected to display this tendency particularly as they have the lowest classification score, and hence presumably the least trunk function.

Future work should focus on improving on-court assessments, including other key performance factors such as agility and ball handling for each set-up. An optimal wheelchair set-up will benefit as many performance factors as possible, with player and coach discussions crucial in establishing the most relevant factors for each individual's on-court performance. Increased analysis of linear sprints, such as monitoring peak acceleration magnitudes and subsequent hand position could also allow for greater understanding of set-up effects. Finally, testing of the effects the theoretical best set-up suggested from this testing approach has on performance is required.

\section{Conclusions}

Optimising the interaction between the user and wheelchair for wheelchair court sports is a difficult process, due to (i) the difficulty in assessing on-court performance; (ii) the variations in trunk, arm, and hand functions across players; and (iii) the trade-offs for specific performance factors. An orthogonal design approach has the potential to investigate a range of set-up factors with reduced on-court testing, improving understanding at an individual level of analysis. This work focused on three case studies for players of various classification scores and the effect SH, SDep, SA, and TP had on $5 \mathrm{~m}$ sprint times and kinematics of the first three strokes. Due to the small sample sizes, lower and upper limits were set using the TE for each parameter to identify meaningful differences. Changes in sprint times were evident for a number of factors, with these factors varying between players. Additionally, whilst some meaningful differences were identified for kinematic measures, further implications of wheelchair set-up may be present. These potential findings are supported by previous research, with decreased SAs resulting in RelAngs further from TDC for two of the three players. This approach has the potential to reduce player testing whilst providing important information on wheelchair set-up at an individual level, however input from individuals experienced in the sport is crucial to ensuring findings are relevant and promote improved on-court performance.

Acknowledgments: The authors would like to acknowledge the support from the Australian Wheelchair rugby team throughout testing, the Australian Paralympic Committee and the Australian Government Research Training Program Scholarship. 
Conflicts of Interest: The authors declare no conflict of interest.

\section{References}

1. Goosey-Tolfrey, V. Supporting the paralympic athlete: Focus on wheeled sports. Disabil. Rehabil. 2010, 32, 2237-2243.

2. Mason, B.; van der Woude, L.H.; Goosey-Tolfrey, V.L. The ergonomics of wheelchair configuration for optimal performance in the wheelchair court sports. Sports Med. 2013, 43, 23-38.

3. Vanlandewijck, Y.; Theisen, D.; Daly, D. Wheelchair propulsion biomechanics: Implications for wheelchair sports. Sports Med. 2001, 31, 339-367.

4. Vanlandewijck, Y.C.; Verellen, J.; Tweedy, S. Towards evidence-based classification in wheelchair sports: Impact of seating position on wheelchair acceleration. J. Sports Sci. 2011, 29, 1089-1096.

5. Mason, B.; van der Woude, L.; Tolfrey, K.; Goosey-Tolfrey, V. The effects of rear-wheel camber on maximal effort mobility performance in wheelchair athletes. Int. J. Sports Med. 2012, 33, 199-204.

6. Mason, B.; Van Der Woude, L.H.; Tolfrey, K.; Lenton, J.P.; Goosey-Tolfrey, V.L. Effects of wheel and handrim size on submaximal propulsion in wheelchair athletes. Med. Sci. Sports Exerc. 2012, 44, 126-134.

7. Vanlandewijck, Y.C.; Thompson, W.R. Handbook of Sports Medicine and Science, the Paralympic Athlete; John Wiley \& Sons: Hoboken, NJ, USA, 2011.

8. Molik, B.; Lubelska, E.; Koxmol, A.; Bogdan, M.; Yilla, A.B.; Hyla, E. An Examination of the international wheelchair rugby federation classification system utilizing parameters of offensive game efficiency. Adapt. Phys. Act. Q. 2008, 25, 335-351.

9. Churton, E.; Keogh, J.W. Constraints influencing sports wheelchair propulsion performance and injury risk. Sports Med. Arthrosc. Rehabil. Ther. Technol. 2013, 5, 1-10.

10. Mason, B.; Porcellato, L.; van der Woude, L.H.; Goosey-Tolfrey, V.L. A qualitative examination of wheelchair configuration for optimal mobility performance in wheelchair sports: A pilot study. J. Rehabil. Med. 2010, 42, 141-149.

11. Usma-Alvarez, C.C.; Fuss, F.K.; Subic, A. User-centered design customization of rugby wheelchairs based on the Taguchi method. J. Mech. Des. 2014, 136, 1-13.

12. Mason, B.; Lenton, J.; Leicht, C.; Goosey-Tolfrey, V. A physiological and biomechanical comparison of overground, treadmill and ergometer wheelchair propulsion. J. Sports Sci. 2014, 32, 78-91.

13. Van der Slikke, R.M.A.; Berger, M.A.M.; Bregman, D.J.J.; Veeger, H.E.J. Wheel Skid Correction is a Prerequisite to Reliably Measure Wheelchair Sports Kinematics Based on Inertial Sensors. Procedia Eng. 2015, 112, 207-212.

14. Melrose, K.C.C. Rhino Script Form: Offensive- High Point. 2014. Cited 2017. Available online: http://www.wheelchairs.co.nz/custommade/rugby.html (accessed on 30 November 2017).

15. Haydon, D.S.; Pinder, R.A.; Grimshaw, P.N.; Robertson, W.S.P. Overground Propulsion Kinematics and Acceleration in Elite Wheelchair Rugby. Int. J. Sport Physiol. Perform. 2017, 1-7, doi: 10.1123/ijspp.2016-0802.

16. Paulson, T.; Goosey-Tolfrey, V. Current perspectives on profiling and enhancing wheelchair court sport performance. Int. J. Sport Physiol. Perform. 2017, 12, 275-286.

(C) 2018 by the authors. Licensee MDPI, Basel, Switzerland. This article is an open access article distributed under the terms and conditions of the Creative Commons Attribution (CC BY) license (http://creativecommons.org/licenses/by/4.0/). 